\title{
Non-Synthetic European Low Voltage Test System
}

\author{
Arpan Koirala, Lucía Suárez-Ramón, Bassam Mohamed, Pablo Arboleya Senior Member, IEEE
}

\begin{abstract}
The use and development of standard test systems has been critical for testing all kind of algorithms, operation procedures and planning purposes in transmission and distribution networks. With the irruption of advanced metering infrastructure based among others on smart meter technology, distribution systems suffered a drastic modernization in many regions of the world. The smart grid concept translated to the terminal distribution systems implies a drastic change of paradigm in the way these kinds of systems have been planned and operated. Although there are several distribution test systems, most of them are synthetic and there are very few test feeders and networks representing the European style distribution system. In this paper, a real (non-synthetic) comprehensive distribution test network representing a typical European town's network is presented. The model also features a provisioning mechanism to incorporate smart meter data obtained from the consumers meter in order to study the distribution network status. This test network will provide a tool that allows researchers to work on algorithms and optimization of resources for European style distribution systems. The test network also demonstrates the impact of neutral voltage in European style distribution networks. Both researchers and the distribution company provide with the raw data extracted from the GIS system and all the functions to convert the data into an OpenDSS model as well as the loads extracted from real smart meters representing 20 days.
\end{abstract}

Index Terms-Distribution system, test network, four-wire system, DSO, Smart Meters, Smart Grid.

\section{INTRODUCTION}

D ISTRIBUTION systems have experienced massive changes since the introduction of smart meters in consumer premises and the modification in the substations. The introduction of small renewable and consumerbased generation has now converted many consumers into prosumers. The decreasing price for storage systems and the presence of intermittent generation resources will force a change in the power distribution paradigm. Future distribution systems will have to be more supply-driven than the current demand-driven systems. In order to meet with this paradigm shift in modern power systems, the current distribution networks should be modified to be more controllable. The Distribution System Operators (DSOs) will have a strong role

Arpan Koirala is with KU Leuven ESAT/Electa \& EnergyVille, Belgium.

Lucía Suárez-Ramón the is Chief of Metering Infrastructure Systems at EDP (Electricidade de Portugal), Spain.

Bassam Mohamed and Pablo Arboleya (corresponding author) are with LEMUR Research Group at the Electrical Engineering Department at University of Oviedo, Spain. email: arboleyapablo@uniovi.es

This work was partially supported by the Spanish Ministry of Economy and Competitivity under grant MINECO-17-ENE2016-77919$\mathrm{R}$ (CONCIALIATOR Energy conversion technologies in resilient hybrid AC/DC distribution networks), by the Government of the Principality of Asturias - Foundation for Scientific and Technical Research (FICYT) under grant FC-GRUPIN-IDI/2018/000241 (Laboratory for Enhanced Microgrid Unbalance Research (LEMUR)) and by EDP España under grant FUO-34418 (Development of a model of an EDP electricity distribution network to be solved statically through a calculation engine based on OpenDSS and analyzed using techniques based on Visual Analytics). in the balancing mechanism of future terminal distribution systems. To enable DSOs to take an active role in power system balance, researchers should develop and test the algorithms in a realistic environment. This is key to the development of future distribution test systems.

One of the hurdles for European researchers is lack of representative feeders or networks representing European style distribution systems. The majority of existing test feeders are representing the North American style distribution systems. Basically, in Europe, each power transformer connected to the MV network creates a fully functional LV distribution network with 4 wires supplying a large number of customers or loads. On the other side in most of the cases in U.S. there is not such LV distribution terminal network since distribution is mostly done in MV and a huge number of single-phase transformers supply a single or small number of customers. In Fig. 1 the two different approaches are represented schematically, on the left the American system can be observed, the European is represented on the right.

The only available IEEE European Low Voltage Test Feeder does not clearly represent the European style of distribution network since the neutral wire is not explicitly represented [1] and many of the problems derived from load unbalances cannot be reproduced. Apart from that, it is based on a typical urbanisation model dominated by single-family dwellings, when in reality more than $80 \%$ of the European population lives in residential buildings in urban environments [2]. The need for a real European distribution system in which researchers can study the impact of new technologies is a critical task that should be urgently accomplished.

In this paper, a real (non-synthetic) low voltage European Test Network is presented which has 30 substations with 10290 buses and 8087 loads grouped in 1138 power supplies. We use the term power supply to refer to a group of loads, for instance, a residential building containing a number of different consumers/loads. The loads have time series data extracted hourly from real smart meters which represent a
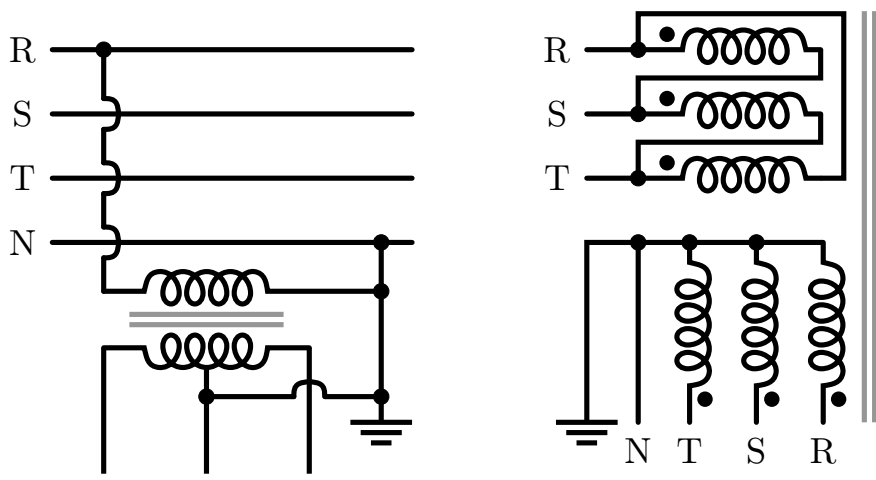

Figure 1: American versus European distribution scheme. American in the left and European in the right 
20 days time span. All lines in terminal distribution are modeled using four wires with the neutral being grounded at transformer neutral only, according to the TT grounding scheme [3]. LV feeders have the capability of changing their connectivity by circuit-breaker operation to modify the LV network connectivity. The size of the network and nature of the loads makes it a unique representative feeder that will provide an opportunity to develop and test different kinds of software. It also provides data for working in distribution system optimization and state estimation techniques providing DSOs with better tools for the future distribution systems.

\section{NEED FOR NEW EUROPEAN TEST SyStem}

In 1991, the first set of four distribution test feeders was published by IEEE Test Feeder Working Group [4]. It had four North American style Distribution feeders with an unbalanced load and untransposed network. The fifth test feeder was proposed in [5] in 2001. In 2009, the requirement of new test feeders was motivated in [6] in seven different areas and consequently, new test feeders were developed. The different test feeders created to address the roadmap were neutral to earth voltage (NEV) test feeder [7], 8500 node test feeder [8], Comprehensive Distribution Test Feeder [9] and low voltage network test system [10]. There are other distribution test feeders representing the North American style network presented as part of EPRI's Green Circuit Project Database and PNNL Taxonomy Feeder Study [11]. The EPRI set of test feeders consists of 6 different real feeders sanitized for public use while the PNNL test feeder has 24 representative radial distribution feeders based upon statistical analysis of 575 distribution feeders. Though many works have been done in the sector of North American style distribution feeder, very limited studies have been done in the sector of European style distribution system. IEEE has currently only one European test feeder, the so called "European LV test feeder" [12] published in 2014 but it does not completely represent the European style apart from maintaining the base frequency of $50 \mathrm{~Hz}$ and LV maintained at $416 \mathrm{~V}$. There are few independent works to generate test feeders representing specific regions like [13] for Western Australia, [14] for North England and [15] for Europe, but they have various limitations. The representative Western Australian feeders have $9 \mathrm{MV}$ and $8 \mathrm{LV}$ feeders created using a clustering algorithm to include the mean feature of distribution feeders. The representative North England feeder has 11 representative feeders to represent the different type of feeder in North England. All of them act as individual feeders and not as the combination of feeders which limits their usage. The European Representative Network is a robust example based on the data of 79 utilities, but the final network is synthetic.

\section{A. Limitation of European Low Voltage Test Feeder}

The inclusion of low voltage feeder at $416 \mathrm{~V}$ and $50 \mathrm{~Hz}$ makes the IEEE European LV test feeder get quite close to the a European style distribution grid. The per-minute load data series makes it suitable for studies of control and economic aspects of the distribution system. Still, much additional work is required to represent an actual European style distribution system.

1) Neutral Grounding: In the test feeder, the four-wire model of the European style distribution system is reduced to a three-wire equivalent system by using Kron's reduction in Carson's equation[16]. This reduction is based on the assumption that the neutral conductor is multiple grounded and the consumer neutral and earth ground is common. It assumes that the neutral current returns to the source through the ground. This might give the correct result in case of Medium Voltage (MV) lines and systems with shorter LV lines. But European TT low voltage distribution system has very long LV lines with isolated neutrals. The neutral supplied to the consumer is separated from the consumer ground. Kron's reduction assumes that the neutral is grounded at every node and gives a wrong interpretation of the consumer end voltage. In the isolated neutral case, all neutral current returns to the distribution substation transformer neutral where it is grounded. In this process, the current flowing through the neutral causes a voltage drop which appears as the neutral voltage which is always above the assumed $0 \mathrm{~V}$ neutral Voltage and becomes higher based on the distance from the substation and existing unbalances in the feeder. The voltage thus received by the consumer will be phase-to-neutral voltage instead of phase-to-ground voltage as assumed by the model.In [17], the system was modified removing the three wire equivalent and adding a more realistic four-wire system, since it was important to evaluate neutral currents and voltage.

2) Time series load: The load in IEEE European is time series format with 1440 load data representing every minute of the day. The resolution in this case is acceptable since the smart meters connected through power line communication (PLC), providing values each 15 minutes or hourly [18]. In other cases like for instance, the work presented in [19], the data are fed into the IEEE European Low Voltage Test Feeder each second using the loads obtained from ADRES data base [20].

3) Feeder Type: The usual European LV network consists of distribution transformers with a capacity of 100 to $1000 \mathrm{kVA}$. Each transformer has multiple feeders emanating from the substation and a few feeders of other substations terminating at that substation to provide $n-1$ reliability. They are connected through circuit breakers with the status of Normally Open (NO) or Normally Closed (NC). The test feeder is a single feeder connected to a transformer which has no possibility of changing the LV network structure, thus providing very limited flexibility. Further, the impact of this feeder in the transformer is the impact of an individual feeder, the impact of multiple feeders in the same transformer as present in a usual European network has not been considered.

4) Load Type: In European LV distribution system, there are both single phase and three phase loads. The three phase load can be balanced or unbalanced. Each feeder consists of 40 to 60 loads on average. The European LV test feeder consists of 55 single phase loads only. The number of loads matches with the real condition but all of them being single phase is quite unrealistic. Further, the 0.8 MVA transformer is loaded at $10 \%$ of capacity which is very low compared to the general 
norm of loading distribution transformers is around $70 \%$ in peak load.

\section{Real (Non-Synthetic) European Test Network}

Test feeders and networks of the distribution systems are created basically in 4 ways. The first most common way is taking the data from the real distribution network, removing the private data and sanitizing it with new easy names. The second method clusters several actual networks to build a synthetic network. The third method consists in the manual design of the distribution network. The fourth method is based on planning tools using economic and technical criteria in order to create a realistic distribution networks [21]. In our case, the European LV test network is generated in accordance with the first approach by taking the real data of a city and sanitize it for removing the private data and redundant information in GIS data. The network model is then created in OpenDSS.

\section{A. Introduction to Test Network}

As discussed in the previous section, the majority of the current test feeders and networks are based on the North American style. The existing European LV test feeder is incomplete and does not represent the actual European style distribution network in terms of inclusion of neutral voltage due to TT grounding in substation neutral and isolated neutral of the consumer, load type, integration with smart meter data and inclusion of multiple LV feeder in a substation.

With the increasing Distributed Energy Resources (DERs) penetration in distribution networks, DSOs need a tool to assess the impact of these new agents in the whole network more efficiently. The development of realistic test networks is an essential task.

The basic features of the proposed network are:

- The rated voltage is $416 \mathrm{~V}$ (phase-to-phase) and the frequency $50 \mathrm{~Hz}$.

- The LV network is designed as a 4-wire system with isolated neutral from consumer ground. Neutral voltage can be monitored for each bus and neutral is grounded at substation only.

- The test network consists of 8087 loads and 30 distribution transformers representing a small town.

- There is a total of 10290 buses of which 2681 buses are monitored.

- The load data is defined as a time series consisting of hourly smart meter data within a 20 days interval.

In the next sections, a brief description of the network and its components will be presented. The whole data set and functions will be attached to the paper as supplementary material. It will be possible to modify the topology of the network changing the data obtained from the GIS system and then compute the OpenDSS automatically with the functions provided by the authors.

\section{B. Network Description}

Fig. 2 represents the single line diagram of the proposed European distribution test network. The red circles represent the distribution substations and the black lines represent the LV network. The MV lines are not considered in this model, assuming that all substations are supplied by a single infinite source. The different components of the network are discussed below:

1) Voltage Source: The source is defined by the short circuit current in three phases and one phase similar to the IEEE European test feeder. The base voltage is $22 \mathrm{kV}$. All the substations are connected to a single nearly infinite source.

2) MV lines: Virtual MV lines connect the source with the transformers. They are designed to have a very low resistance of $25 \mu \Omega$ to minimize the impact of MV lines, but this value can be adjusted depending on the short circuit power in the different points of the network. MV lines are a three wire-system connected to the delta side (primary) of the transformers. There are 30 such lines, one per transformer substation. They are named mv1, mv2, .. mv30 and the assigned line code is 101 with a length of $5 \mathrm{~m}$ (see Table I).

3) Distribution Transformers: There are 30 distribution transformers, all of them with the same delta-wye (grounded) configuration. The voltage rating is $22 / 0.420 \mathrm{kV}$ and there are three differnt power ratings 100, 250 and 630kVA. The impedance of the transformer is $4 \%$ for each unit. Table II contains the description of two power transformers. It must be mentioned that we modeled the whole system using OpenDSS. In the case of power transformers we used a very simple model considering only the percentage reactance of the primary and secondary circuits, the parameter XHL. OpenDSS Manual containing the power transformer mathematical model can be found in [22]. As it can be observed in the page 124 of the manual, there is many available parameters for defining the power transformer in a very deep way. The reader can edit the model provided as an additional material and use whatever other transformer parameter that consider necessary for any specific study.

4) Feeder: We define feeders as the elements that connect the transformer secondary with the circuit breaker in the substation. All feeders are modeled as 4-wire elements with the line code $205\left(3(1 \times 240) 1 \times 150 \mathrm{~mm}^{2}\right)$. In the GIS data we can find feeders with open and closed circuit breakers. Only the ones with the closed circuit breaker are added to the electrical model. In the case of study we have 161 feeders named as feeder 1 , feeder $2, \ldots$, etc. (See an example of how feeders are defined in Table III).

Table I: MV lines in test network

\begin{tabular}{cccccc}
\hline MV Line name & Bus 1 & Bus 2 & phases & Linecode & Length (m) \\
\hline mv2 & Source & 2 & 3 & 101 & 5 \\
mv3 & Source & 3 & 3 & 101 & 5 \\
\hline
\end{tabular}

Table II: Distribution transformer in test network

\begin{tabular}{cccccc}
\hline DT name & [Bus1 Bus2] & Connection & KVA & kVs & XHL \\
\hline TD401346 & {$[131]$} & [Delta Wye] & 630 & {$[220.420]$} & 4.0 \\
TD400291 & {$[2$ 32] } & [Delta Wye] & 1000 & {$[220.420]$} & 4.0 \\
\hline
\end{tabular}




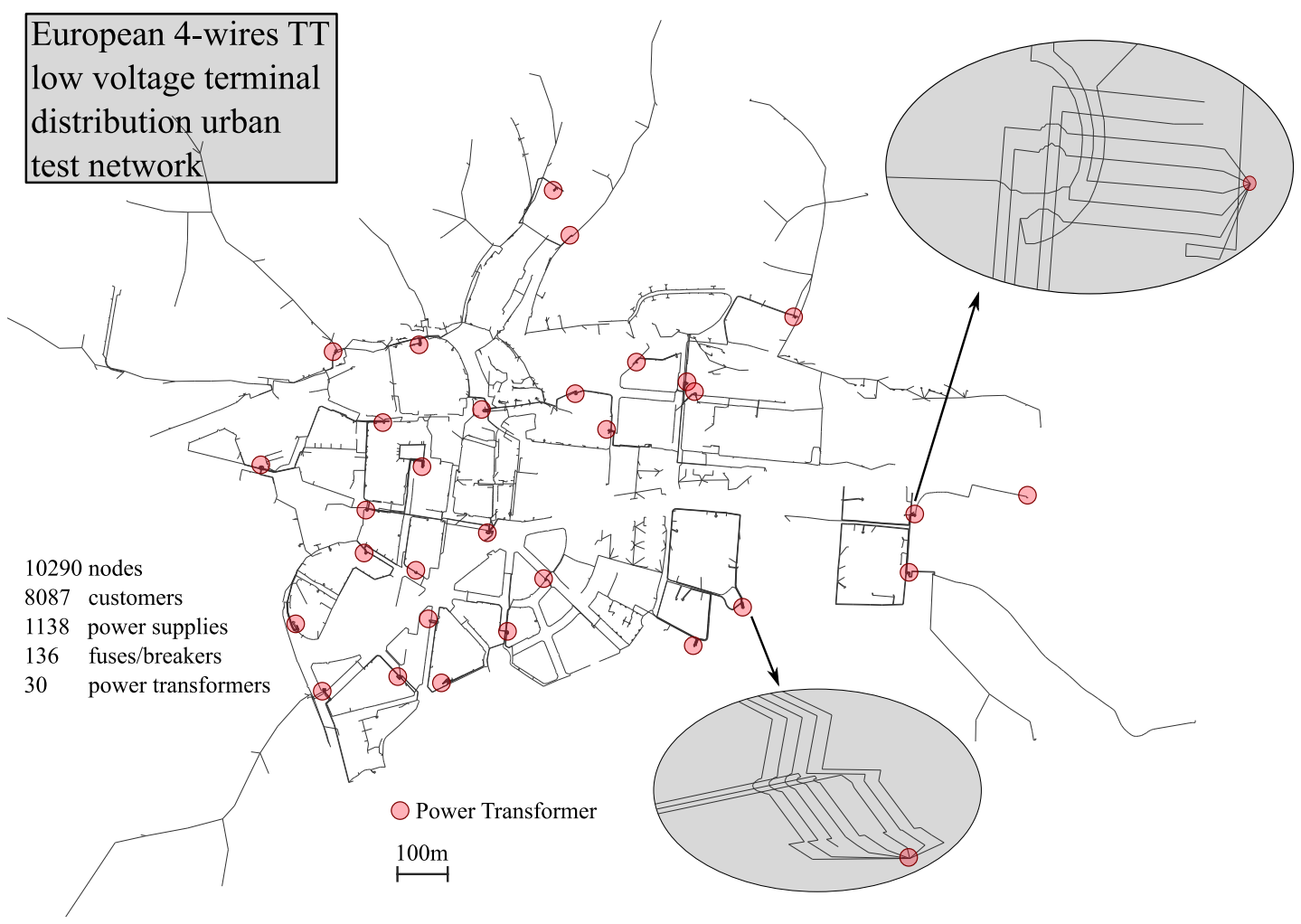

Figure 2: Single line Diagram of the European 4-wires TT low voltage terminal distribution test network

Table III: LV feeders in test network

\begin{tabular}{|c|c|c|c|c|}
\hline Feeder name & [Bus1 Bus2] & phase & Linecode & Length (m) \\
\hline feeder1 & [31 31_1] & 4 & 205 & 4.340 \\
\hline feeder2 & {$\left[\begin{array}{lll}31 & 31 \_2\end{array}\right]$} & 4 & 205 & 3.678 \\
\hline
\end{tabular}

Table IV: Substation circuit breaker in test network

\begin{tabular}{ccccc}
\hline Feeder name & [Bus1 Bus2] & phase & Linecode & Length $(\mathrm{m})$ \\
\hline cktbk1 & {$\left[\begin{array}{ll}31 \_1 & 2643\end{array}\right]$} & 4 & 102 & 0.5 \\
cktbk2 & {$\left[\begin{array}{ll}31 \_2 & 2644\end{array}\right]$} & 4 & 102 & 0.5 \\
\hline
\end{tabular}

Table V: Load definition in test network

\begin{tabular}{llllllll}
\hline Name & Phases & Bus & RYB & kV & kW & PF & loadshape \\
\hline LOAD1 & 1 & 1233 & B & 0.23 & 1 & 0.95 & Shape_1 \\
LOAD7 & 3 & 1240 & RYB & 0.4 & 1 & 0.95 & Shape_7 \\
\hline
\end{tabular}

Table VI: Neutral reactor definition in test network

\begin{tabular}{llllll}
\hline Name & Phases & Bus1 & Bus2 & $\mathrm{R}(\Omega)$ & $\mathrm{X}(\Omega)$ \\
\hline grnd1 & 1 & 31.4 & 31.0 & 5 & 0.01 \\
grnd2 & 1 & 32.4 & 32.0 & 5 & 0.01 \\
\hline
\end{tabular}

5) Substation Circuit-breaker: Each feeder has a circuit breaker which connects it with LV network. Only the breakers which are closed are considered in this test network. The effective resistance of the circuit breaker is assumed to be $25 \mu \Omega$ and is represented by line element with line code 102 and length $0.5 \mathrm{~m}$ (See table IV). Each feeder has one circuit breaker making 161 breakers in total named as cktbk1, cktbk2, etc. The Bus 1 of the circuit breaker is connected to the feeder while Bus 2 is connected to the LV network.
6) LV Network: The LV Network connects the transformer to the consumers. The LV network is modeled as a set of 4wire lines. The neutral wire is isolated. The line code for the different type of cable is presented in appendix A and the respective resistance and reactance (in $\Omega$ ) of the cross-section is represented in appendix B. The resistance and inductance of a specific line are represented by $4 \times 4$ matrices. In case of the matrices below (representing lines with the code 201) only the self-resistance and self-inductance has been considered.

$$
\begin{aligned}
\text { Rmatrix_201 } & =\left[\begin{array}{cccc}
1.23 & 0 & 0 & 0 \\
0 & 1.23 & 0 & 0 \\
0 & 0 & 1.23 & 0 \\
0 & 0 & 0 & 1.23
\end{array}\right] \\
\text { Xmatrix_201 } & =\left[\begin{array}{cccc}
0.08 & 0 & 0 & 0 \\
0 & 0.08 & 0 & 0 \\
0 & 0 & 0.08 & 0 \\
0 & 0 & 0 & 0.08
\end{array}\right]
\end{aligned}
$$

There is a total of 2490 line segments. But to define the physical coordinates of all points within a line and create a graphical topology similar to the real one, the segments are split into several parts. These segments are named as sub-parts of the main segment and only the last sub-part is named by an integer number and is monitored. Bus 2 of the last sub-part matches with the name of the line to make monitoring easy. The names of the line segments start from 61. The first 60 integers are used to represent $\mathrm{HV}$ and $\mathrm{LV}$ sides of distribution transformers. Figure 3 represents the way in which the different components of the system are represented. The name in red is the name of line segments 


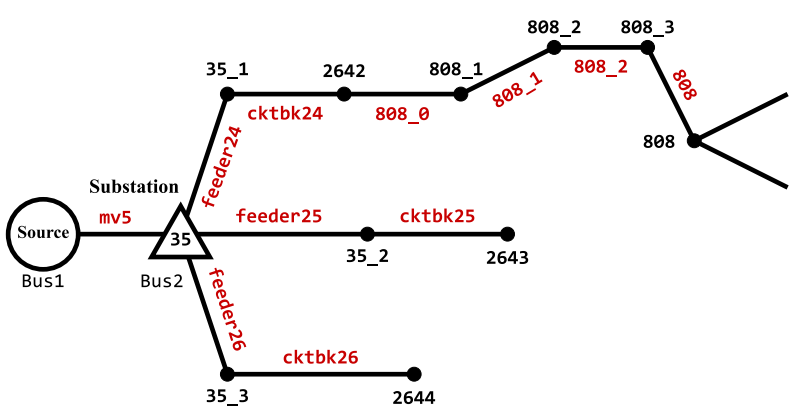

Figure 3: Bus indexing representation in a substation.

while that in black is the bus number. The reason for this nomenclature is to reduce redundant buses in the test feeder for monitoring purpose [23] and for maintenance of same bus and line numbers when LV configuration is changed.

7) LV Network Breakers: A special feature of these LV test system is the presence of breakers embedded in the LV network which can be operated in coordination with other breakers in the substations to change the network configuration. The effective resistance of these circuit breakers is assumed to be $25 \mu \Omega$. The OpenDSS file only contains those breakers which are closed and they are modeled by line element with very low impedance. The breaker elements are represented as lines with name fusel, fuse 2 , ...etc and their parameters are similar to the substation circuit breakers defined in Table IV.

The open breakers are commented in the OpenDSS script. If there is a change in the network produced by a switching operation of one or more breakers, the MATLAB script is developed for generation a new OpenDSS file. The switching can be done by changing the Excel file obtained from GIS representation of the network.

8) Load: The load is defined by the number of phases, a load shape and, in case of single phase, the connected phase. The neutral of the load is connected to the distribution network neutral and is different from the consumer ground. The system is designed in a way that the consumer voltage is the phase to neutral voltage instead of the phase to ground voltage. Each load is connected to a load shape with 20 days of hourly data. The load data is obtained from the actual smart meter on the consumer premises. The load is modeled as constant PQ load with P given through the load shape and a constant power factor of 0.95 . The users can change the power factor in an easy way editing the model or adding active and reactive data, they can also change the consumption files or add PV generation to the buildings or consumers since the model is open and flexible. There are 8087 loads grouped in 1138 power supplies in the test network proposed which are a combination of single phase and three phase loads. The load shape for each load is extracted from the actual smart meter data. We would like to remark that the load shapes are exactly those obtained from the readings of the real smart meters, no curve fitting or parametrization has been done. All load data are provided as additional material and we think can be a very good material for other authors studying techniques for load shaping, parametrization, pattern extraction, customers segmentation, etc.
9) Reactor: The reactor component is used for grounding the distribution substation neutral. A reactor of resistance $5 \Omega$ and reactance of $0.01 \Omega$ is used in every substation on the $\mathrm{LV}$ side for grounding the transformer neutral.

\section{Meters and Monitors}

To observe the electrical variables in the substations and the LV network, the monitor models were added to the mathematical model. The monitors are divided into 3 types, MV lines, feeder and LV lines monitors. They are used for monitoring active power, reactive power, voltage and current in the MV lines, LV feeder origin and monitored LV segments. Since the model is 4-wire, we can obtain the neutral voltage and current in a direct way. The MV network is a 3-wire system with no physical neutral. The meter elements define the energy meter for every substation on the MV side. The complete LV network of a specific substation is defined in a single control zone in OpenDSS.

\section{Model Generation Script Description}

One of the main purposes of the project described in this paper is to provide a toolbox which enables us to directly convert the raw GIS data into an OpenDSS model with its script ready to be solved. In this section, we first describe the raw data, then the script used to convert the raw data to an OpenDSS file and a simple script to run power-flow analysis and post-analysis in Matlab.

\section{A. Explanation of raw data [GIS_data]}

The network raw data consists in the data obtained from the GIS file proceeding from Bentley GIS and the load data obtained from smart-meter readings. This raw data is saved in four Excel files to the GIS_data folder, namely: 'master.xlsx', load.xlsx', 'phase meters.xls' and filey.xlsx'. The master.xlsx' is the network raw file with six sub-files (six different sheets inside the Excel workbook) underneath explained below:

1) Transformer (CT -TRAFO): Table VII represents the raw file for the transformers. The transformers name is uniquely represented in column $\mathrm{H}$ which is taken as transformer name in the test network. The columns $\mathrm{F}$ and $\mathrm{K}$ represent $\mathrm{MV}$ and $\mathrm{LV}$ side of the transformer respectively. The column $\mathrm{J}$ represents the capacity of the transformer in $\mathrm{kVA}$ and columns $\mathrm{D}$ and $\mathrm{E}$ are the $\mathrm{X}$ and $\mathrm{Y}$ coordinates of the substation.

2) Feeder (Linea BT): The feeder data in the raw file obtained from the GIS are given as shown in table VIII. This file has information of all feeders connected to the transformers with the status of the sub-station circuit breaker. The columns I and $\mathbf{J}$ represent the normal state and the current state of the circuit breaker. To simulate any change in the network, the current state of the circuit breaker should be changed. The symbol 'A' refers to open breaker and ' $C$ ' refers to the closed breaker' ${ }^{1}$.

\footnotetext{
${ }^{1}$ Abierto and Cerrado in Spanish means Open and Closed respectively
} 
Table VII: Raw file for transformer data from the GIS

\begin{tabular}{|c|c|c|c|c|c|c|c|c|c|c|}
\hline A & B & $\mathrm{C}$ & $\mathrm{D}$ & $\mathrm{E}$ & $\mathrm{F}$ & $\mathrm{G}$ & $\mathrm{H}$ & I & $\mathrm{J}$ & $\mathrm{K}$ \\
\hline MSLINK & CLAVE_BDI & DES & $\mathrm{X}$ & $\mathrm{Y}$ & MSLINK CELDA & CLAVE CELDA & CLAVE TRAFO & DES TRAFO & POT. TRAFO & NUDO CELDA TR. \\
\hline 65043 & $\mathrm{C} 000601$ & CT XXX & 284345.89 & 4807588.1 & 65045 & PO006217 & TD401346 & TRAFO XXX & 630 & 3865289 \\
\hline
\end{tabular}

Table VIII: Raw file for feeder data from the GIS

\begin{tabular}{|c|c|c|c|c|c|c|c|c|c|}
\hline $\mathrm{A}$ & B & $\bar{C}$ & $\mathrm{D}$ & $\mathrm{E}$ & $\mathrm{F}$ & $\bar{G}$ & $\mathrm{H}$ & I & $\mathrm{J}$ \\
\hline Mslink & Linea & Mslink CT & Clave CT & NO & $\mathrm{NE}$ & $\mathrm{X}$ & $\mathrm{Y}$ & EST_normal & EST_operac \\
\hline 775431 & 1 & 65043 & $\mathrm{C} 000601$ & 3865289 & 108023 & 284346.6 & 4807583.8 & $\mathrm{C}$ & $\mathrm{C}$ \\
\hline
\end{tabular}

Table IX: Raw file for line segment data from the GIS

\begin{tabular}{cccccccc}
\hline A & B & C & D & E & F & G & H \\
Mslink & Longitude & Tipo & Mslinea & Clave linea & Nudo origen & Nudo destino & Tipo Cable \\
\hline 1374736 & 1 & Subterraneo & 73796 & 5 & 41291 & 3225463 & BT-RZ0,6/1 KV 4*25 KAL \\
\hline
\end{tabular}

Table X: Raw File for Line Coordinates data from the GIS

\begin{tabular}{cccccccc}
\hline A & B & C & D & E & F & G & H \\
MSLINK & Longitude & Tipo & MSLINK Linea & CLAVE Linea & Orden Vertice & X & Y \\
\hline 74242 & 34 & Aereo & 74239 & 1 & 0 & 284321.44 & 4807668.4 \\
\hline
\end{tabular}

Table XI: Raw file for LV network circuit breaker data from GIS

\begin{tabular}{ccccccc}
\hline A & B & C & D & E & F & G \\
Mslink Fusible & X & Y & Nudo Origen & Nudo Extremo & Est. Normal & Est. Operac \\
\hline 80034 & 284481.72 & 4808081.4 & 44257 & 1402524 & A & A \\
\hline
\end{tabular}

Table XII: Raw file for load curve data

\begin{tabular}{|c|c|c|c|c|c|c|}
\hline $\mathrm{C}$ & $\mathrm{D}$ & $\mathrm{E}$ & $\mathrm{F}$ & $\mathrm{G}$ & $\mathrm{H}$ & I \\
\hline Referencia & Fecha & Dia & Estacion & Activa E & Activa S & Reactical \\
\hline SAG0145432047 & $2 / 26 / 2018 \quad 1: 00$ & $2 / 26 / 20180: 00$ & $\mathrm{~W}$ & 64.505 & 0 & 7.39 \\
\hline
\end{tabular}

Table XIII: Raw file for LV network links with the loads

\begin{tabular}{cccccc}
\hline A & B & C & D & E & F \\
Mslink & Clave BDI & Nudo Orig & Nudo Dest & X & Y \\
\hline 1220006 & 15152501 & 954350 & 3790730 & 284846.86 & 4807846.4 \\
\hline
\end{tabular}

Table XIV: Raw file for smart meter in the network

\begin{tabular}{|c|c|c|c|}
\hline$\overline{\mathrm{A}}$ & $\mathrm{R}$ & $S$ & $\mathrm{~T}$ \\
\hline Referencia & Acometidas & Linea & Clave $\mathrm{Ct}$ \\
\hline SAG0145432047 & 36767901 & 2 & $\mathrm{C} 000601$ \\
\hline
\end{tabular}

Table XV: Raw file for phase of smart meter

\begin{tabular}{cccccc}
\hline A & B & C & D & E & F \\
ID & Fase (phase) & Linea & Mediada & Ultimo Estado & Ct \\
\hline SAG0145432047 & R & TF1CB & OK & Active & CT002051 \\
\hline
\end{tabular}

Column E refers to the LV side of the transformer while column $F$ refers to the starting node for the feeder. In the OpenDSS model, the feeder and circuitbreaker are represented by line elements and the state is omitted if the breaker is open. Currently, since the OpenDSS engine does not have an easy interface for network reconfiguration, the Excel file can be modified to update the change in the network OpenDSS model. The important part is to detect the corresponding breaker in the network to be operated to prevent the islanding of the load or part of network.

3) Line Segment (Segmento BT): The line segment file has the data of all line segments in the network (Table IX). The columns $\mathrm{F}$ and $\mathrm{G}$ of this file represent bus 1 and bus 2 of each line segment. Column B represents the length of the segment while column $\mathrm{H}$ is used to represent the wiry type and size. Column $\mathrm{C}$ represents the nature of cable, aerial or underground. The structure of this file is quite complex since bus 1 and bus 2 are not arranged in proper order which should be considered during indexing and model formulation.

4) Line Coordinates (Coordenadas Segmentos): The line segments need coordinates to be represented in a map. The line segments are not always straight, requiring several coordinate points to represent them correctly as shown in table $\mathrm{X}$. These coordinates are represented by defining different lines as shown in Fig. 3 with non-integer names so that monitoring is not done for every coordinate in the segment. Column D of this file represents the line to which the coordinates belong. Column $\mathrm{F}$ shows the order of the specific coordinates in the segment and columns $\mathrm{G}$ and $\mathrm{H}$ are the $\mathrm{X}$ and $\mathrm{Y}$ coordinates to represent the line segment.

5) Circuit Breakers (Fusible): The circuit breakers represent the breakers in the LV network and the snapshot of the raw file obtained from the GIS system is shown in table XI. The file contains details for Bus 1 and Bus 2 in column D and E respectively. Columns $\mathrm{F}$ and $\mathrm{G}$ represents the normal status and current status of the circuit breaker. In order to change the network configuration, the current status of the breaker should be changed. As in previous circuit breakers, $\mathrm{C}$ represent $\mathrm{NC}$ and A represents NO status. This file also establishes links between sub-networks separated by the breakers during the indexing process.

6) Load Connection Points (Acometidas): This is the 
raw file linking the network to the load as shown in table XIII. All loads are connected through the load connection point (Acometida) shown in column $\mathrm{D}$, while the bus in column $\mathrm{C}$ refers to the network bus. For simplicity, in the simulations the load is assumed to connect directly to the network, instead of the load connection points. Columns $\mathrm{E}$ and $\mathrm{F}$ define the coordinates for those load connection points.

The second Excel file containing GIS_data is 'load.xlsx' and its data format is shown in table XIV. The file has a unique smart-meter number in column $\mathrm{A}$ and the load connection point in column R. Column $\mathrm{S}$ determines the transformer to which the smart meter is connected in normal operation. The third Excel file is 'phase meters.xls' and contains the data of the phases to which the smart meter is connected. The phase data was collected from the field verification of results obtained from PLC communication. As shown in table $\mathrm{XV}$, column $\mathrm{A}$ is the unique ID of the smart meter and column $\mathrm{B}$ is the phase to which it is connected. The $\mathrm{R}, \mathrm{S}$ and $\mathrm{T}$ values in column $\mathrm{B}$ indicates phase A, B, and C respectively for single-phase load, while RST represents 3 phase loads. The fourth file/files are 'fileY.xlsx', where $Y=1,2,3, \ldots$ depending upon the load data. These files have the hourly load data obtained from the smart meters. As shown in table XII, column C represents the unique Id of the smart meter and column $G$ contains the active power obtained for that meter. Column $\mathrm{D}$ represents the date and time of the load consumed and column $\mathrm{E}$ is the day and time in which the load data has been retrieved. Due to communication problems or meter change due to failure detection, the load data may be incomplete for a few meters. Column I contains the reactive power of the load but, it is neglected for simplification in the current model since a constant power factor is used. The real values of reactive power could be incorporated in future versions. The rest of the columns is not used in the current model and hence not explained.

The network data files obtained from the real GIS system and the advanced metering infrastructure system can be extracted and converted into the OpenDSS format for power flow calculations using the following functions (also provided as additional material). The two Matlab commands used for creating the OpenDSS file are A_MakeNet.m and B_MakeLoad.m.

\section{B. Conversion module for network}

'A_MakeNet.m' is used to prepare the OpenDSS file and save it to the folder 'RunDss'. The functions used are briefly discussed below:

1) ReadGis.m: This function is meant for extracting data from the Excel file in MATLAB. The function has three variables, 'file_master' (Network file), 'file_load' (Smart Meter File) and 'file_phase' (Load Phase File) to provide the file names of the raw files. These files pointers are used to access those Excel files and their sub-files.

2) linecode.m: This function creates the linecode.txt file which contains linecode of all type of lines described in Line Segments. The line code is generated in $4 \times 4$ format primary impedance matrix with no mutual impedance.

3) bus_cord_modified.m: is used for indexing the bus name. Indexing rules were shown in figure 3 . This code is run twice, before and after seg_crazy.m. The second run is used for matching the line name and Bus 2 name for easy monitoring.

4) Seg_crazy.m: This function is meant for re-arranging the wrongly placed bus. It reshuffles the origin and destination buses in the line segments where it is incorrect.

5) bus_cord_indexed.m: generates the bus coordinates of all the LV buses based on the coordinates in the raw data file and the name according to the new indexing.

6) source_ind.m: is to generate the virtual MV lines in the model.

7) Iine_indexed.m: This file is meant for generating LV line details including the substation breaker and LV feeder. At the same time, it also generates a monitor file for each feeder at the substation and all buses in the LV network.

8) fuse_indexed.m: This function creates the file for all circuit breakers that are not located at the transformers but embedded in the LV network.

9) reactor_neutral.m: This file generates circuit objects used in the neutral grounding.

10) transformer_indexed.m: This function generates the OpenDSS file for transformer components.

11) energymeter.m: defines an energy-meter element file for OpenDSS for every MV line of each transformer.

12) load_indexed.m: This function generates an OpenDSS file for the load element of the network.This function is optional and not included in the usual routine. Specific guidelines to use this function are discussed in appendix $\mathrm{C}$.

\section{Conversion Module for load shape}

'B_MakeLoad.m' is used to create the load-shape file from the smartmeter data extracted in file1, file2, etc. The different functions used for load shape extraction are as follows:

1) save_loadshape.m: This function saves the extracted load-shape file in csv format for the OpenDSS module and is saved to /RunDss/day_20_profile.

2) loadshape_ind.m: It generates an OpenDSS file to link the load element with the loadshape. It gives the file name and location of the respective loadshape. It also provisions for changing the number of load points in the load shape.

3) loadshapeextraction.m: This file checks the smart meter data file and stores it in a vector [shape] according to the indexing rule of load in network extraction module. 


\section{Power Flow Results}

The OpenDSS code thus generated from raw-data is stored in folder /RunDss. The file 'Master.dss' is a default file and is also available in /RunMat/SrcDss.

Power flow simulation can now be done by running C_Run.m in MATLAB. It makes use of the COM interface to do powerflow simulation in OpenDSS engine and stores the final result in /mat/bus.mat. OpenDSS provide two different solvers, the iterative power flow and the direct solution solver. The direct solver manages the loads/generators as admittances and they are merged with the admittance matrix of the system and solved in a direct way. On the other side the iterative solver, loads and generators are injection sources. The iterative solver is provided in two versions, normal current injection solver and Newton solver. The first one is based on the current injection method while the second one is based on the traditional Newton-Raphson method. Iterative current injection is faster and advised for long simulations, it works fine with most of the distribution systems with stiff bulk power source as it is the case that we study. Newton method is a little bit more robust but slower. More detailed information about the algorithms can be found in the OpenDSS manual [? ] and it is beyond the scope of this paper.

The model is designed to monitor the active and reactive power, voltage and current at segment level, feeder level, MV lines (Transformer level) and Network level.

\section{A. Transformer monitoring}

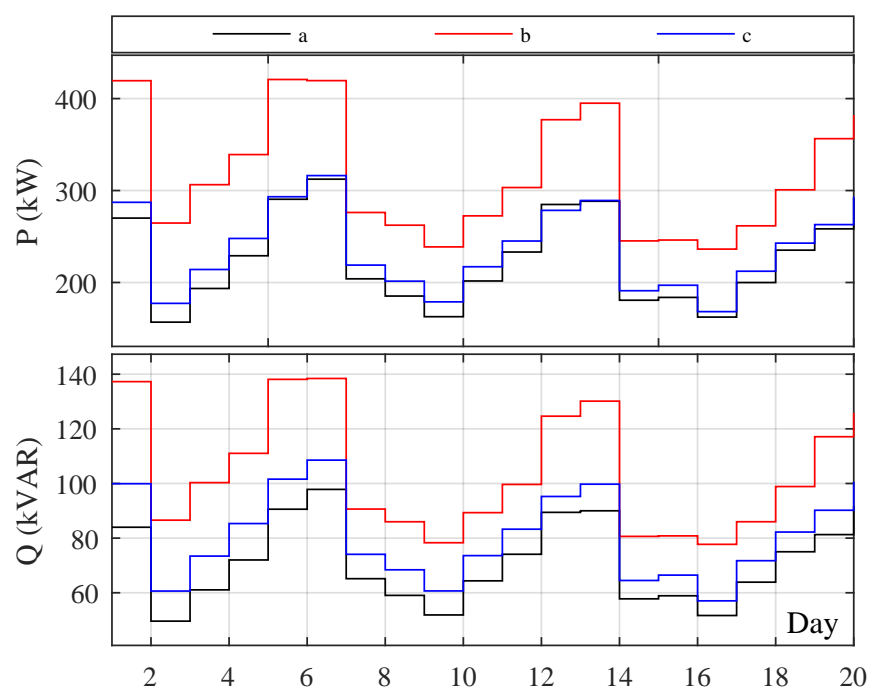

Figure 4: Aggregated power results of Transformer 13 for 20 days

Fig. 4 shows the aggregated active and reactive power flow result of the virtual MV lines connected to transformer 13 for the 20 days. The top plot in Fig. 5 shows the phase voltage of the MV side of transformer 13 for day- 1 in a per hour basis and the bottom plot is the aggregated hourly power in the transformer 13 in day- 1.

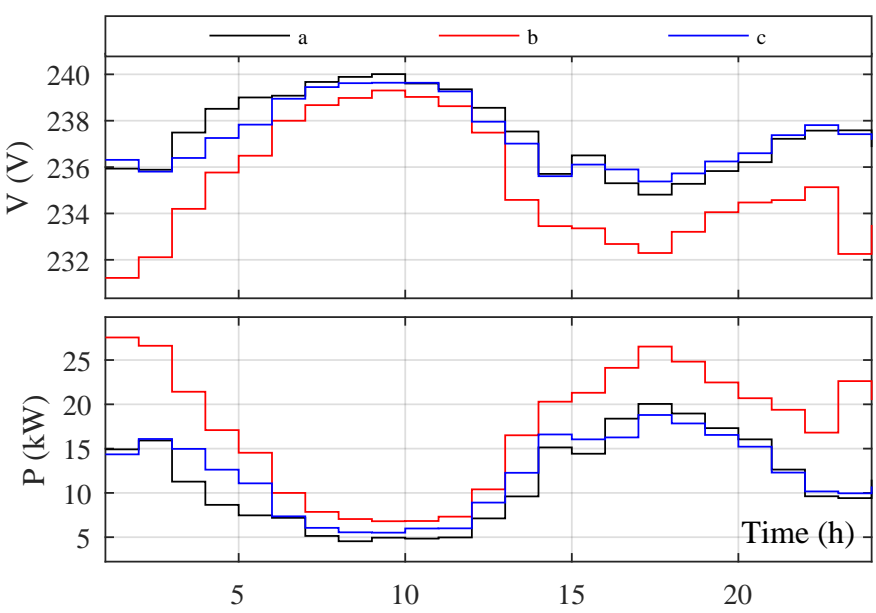

Figure 5: Voltage and power of transformer 13 for 1 day

\section{B. LV segment, feeder and network monitoring}

Fig. 6 represents the output of power flow solution in bus 2113 of the LV network for 20 days. Unlike MV lines, LV lines are 4-wire elements and neutral current and voltage can also be monitored. A separate representation of neutral current and neutral voltage is given. In Fig. 7 we can observe phaseto-ground, phase-to-neutral and neutral-to-ground voltages in bus 2113 during the first day of the study. Most of the previous test networks neglect the neutral or use three-wire equivalents, but in unbalanced European LV networks with TT grounding, neutral voltage plays an important role in consumer voltage. A similar concept is used for feeder monitoring, in which the first bus of the feeder is monitored. Fig. 8 represents the aggregate power in the whole network in $24 \mathrm{hrs}$ in day-1. Similarly, reactive power can be also monitored.

\section{CONCLUSION}

The present paper provides all the required data for simulating a large European style LV distribution network with 4-wire lines and TT grounding system. The authors presented the raw data extracted from a real GIS system of an electrical company and provide all the tools for analysing the raw data and transforming them into a suitable OpenDSS model. There is no such set of data and test system in the literature since all the previous standards are synthetic or contain simplifications that make them not very representative of the European networks. Together with the description of the network, it is also provided the real data on more than 8000 smart meters during 20 days. The whole set constitute a non-synthetic standard test system that may help researchers to make studies like power flow and state estimation studies, test algorithms, assess the impact of distribution generation, prosumers and electric vehicles, planning and monitoring studies, etc. The data provided contain the conventional configuration of the network, however a set of nearly 200 fuses and breakers allow the users to test other configurations. 

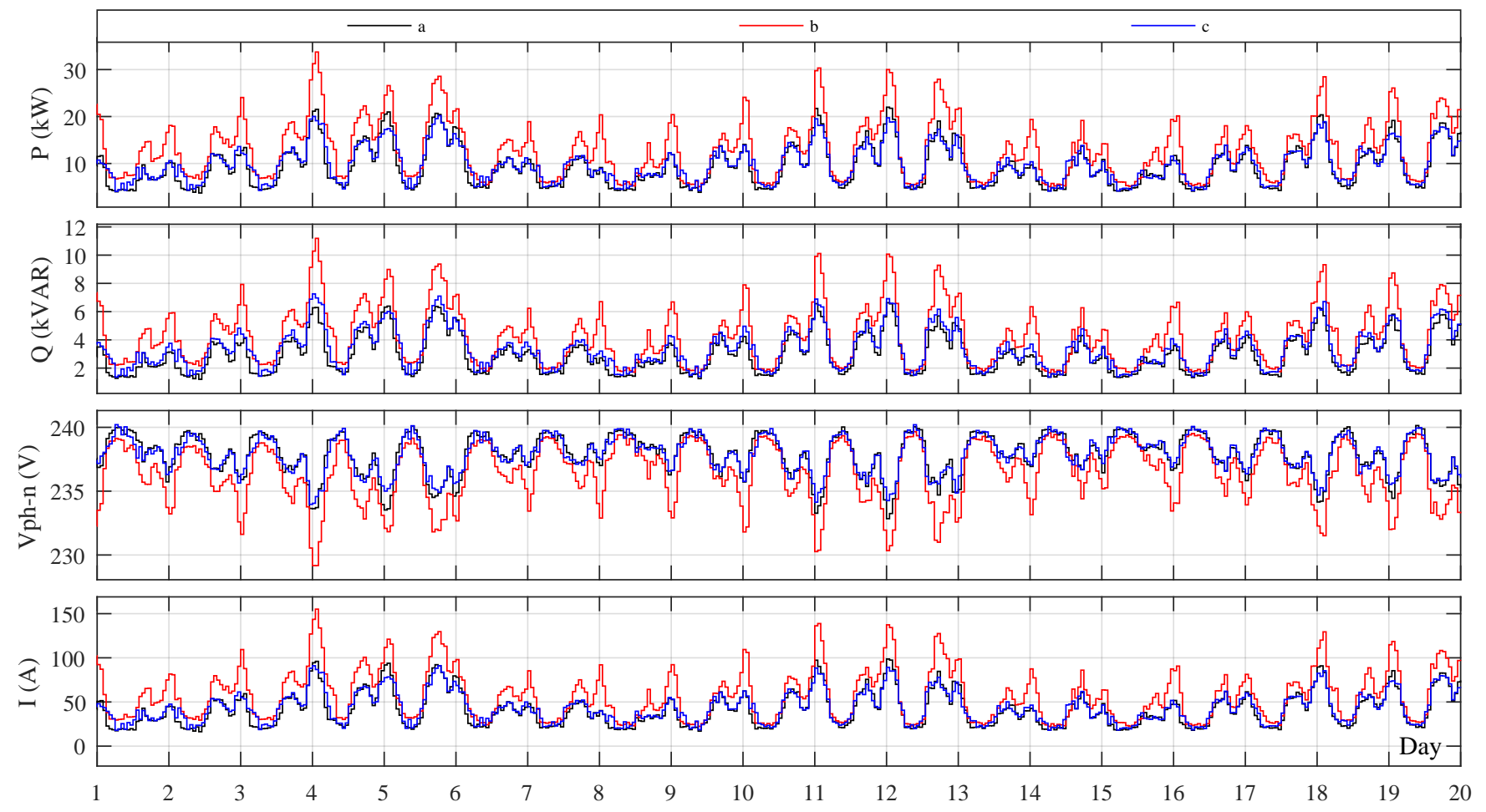

Figure 6: Power Flow result for bus 2113 for 20 days
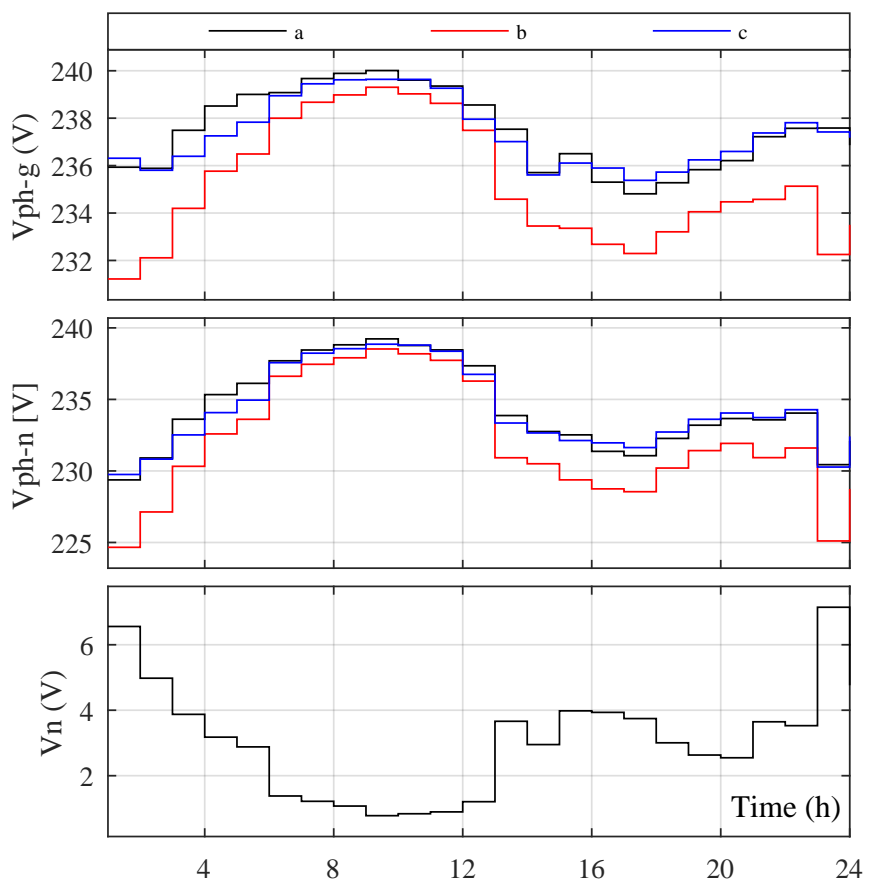

Figure 7: LV voltages of bus 2113 for 1 day

\section{APPENDIX A}

DifFERENT LINE TYPE AND LINE CODE

Table XVI shows the various lines with their respective line codes.

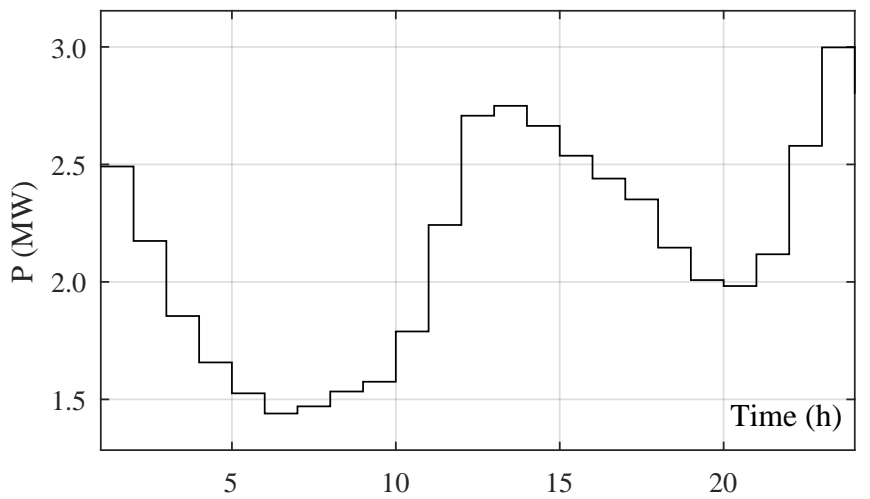

Figure 8: Aggregate power of the whole network for 1 day

\section{APPENDIX B}

\section{Resistance AND IMPEDANCE OF CRoss SeCtion}

Table XVII represents the resistance and reactance (in $\Omega / \mathrm{km}$ ) of different line codes used in the test network.

\section{APPENDIX C}

MANUAL INTERVENTION IN LOAD_INDEXED

The two specific loads have to be commented in file /RunDSS/Load_indexed.txt so that power flow in OpenDSS runs perfectly. The load to be commented are 'Load7990' and 'Load8070'. This is due to some issues with raw data in GIS, most likely a communication failure. A correction is already included in folder/RunMat/SrcDss. 
Table XVI: Line code for different types of lines used along with circuit breaker

\begin{tabular}{lll}
\hline LV Cable Type & Aerial & Underground \\
\hline BT - MANGUERA & 201 & 301 \\
BT - RV 0,6/1 KV 2*16 KAL & 202 & 302 \\
BT - RV 0,6/1 KV 2*25 KAL & 203 & 303 \\
BT - RV 0,6/1 KV 3(1*150 KAL) + 1*95 KAL & 204 & 304 \\
BT - RV 0,6/1 KV 3(1*240 KAL) + 1*150 KAL & 205 & 305 \\
BT - RV 0,6/1 KV 3(1*240 KAL) + 1*95 KAL & 206 & 306 \\
BT - RV 0,6/1 KV 4*25 KAL & 207 & 307 \\
BT - RV 0,6/1 KV 4*50 KAL & 208 & 308 \\
BT - RV 0,6/1 KV 4*95 KAL & 209 & 309 \\
BT - RX 0,6/1 KV 2*16 Cu & 210 & 310 \\
BT - RX 0,6/1 KV 2*2 Cu & 211 & 311 \\
BT - RX 0,6/1 KV 2*4 Cu & 212 & 312 \\
BT - RX 0,6/1 KV 2*6 Cu & 213 & 313 \\
BT - RZ 0,6/1 KV 2*16 AL & 214 & 314 \\
BT - RZ 0,6/1 KV 3*150 AL/80 ALM & 215 & 315 \\
BT - RZ 0,6/1 KV 3*150 AL/95 ALM & 216 & 316 \\
BT - RZ 0,6/1 KV 3*25 AL/54,6 ALM & 217 & 317 \\
BT - RZ 0,6/1 KV 3*35 AL/54,6 ALM & 218 & 318 \\
BT - RZ 0,6/1 KV 3*50 AL/54,6 ALM & 219 & 319 \\
BT - RZ 0,6/1 KV 3*70 ALM/54,6 AL & 220 & 320 \\
BT - RZ 0,6/1 KV 3*95 AL/54,6 ALM & 221 & 321 \\
BT - RZ 0,6/1 KV 4*16 AL & 222 & 322 \\
BT - Desconocido BT & 250 & 350 \\
HV Line 101 & - & \\
LV Circuit Breaker 102 & - & \\
\hline
\end{tabular}

Table XVII: Resistance and reactance (in $\Omega / \mathrm{km}$ )

\begin{tabular}{lllll}
\hline Cross-section & R(Aerial) & X(A) & R(UG) & X(UG) \\
\hline $2 \mathrm{~mm} \mathrm{Cu}$ & 9.9 & 0.075 & 9.9 & 0.075 \\
$4 \mathrm{~mm} \mathrm{Cu}$ & 4.95 & 0.075 & 4.95 & 0.075 \\
$6 \mathrm{~mm} \mathrm{Cu}$ & 3.30 & 0.075 & 3.30 & 0.075 \\
$16 \mathrm{~mm} \mathrm{CU}$ & 1.23 & 0.08 & 1.23 & 0.08 \\
$16 \mathrm{~mm} \mathrm{Al}$ & 2.14 & 0.09 & 2.14 & 0.09 \\
$25 \mathrm{~mm} \mathrm{Cu}$ & 1.34 & 0.097 & 1.538 & 0.095 \\
$35 \mathrm{~mm} \mathrm{Al}$ & 0.907 & 0.095 & 0.907 & 0.095 \\
$50 \mathrm{~mm} \mathrm{Al}$ & 0.718 & 0.093 & 0.718 & 0.093 \\
$54.6 \mathrm{~mm} \mathrm{Al}$ & 0.658 & 0.09 & 0.658 & 0.09 \\
$70 \mathrm{~mm} \mathrm{AL}$ & 0.454 & 0.091 & 0.515 & 0.085 \\
$80 \mathrm{~mm} \mathrm{Al}$ & 0.39 & 0.090 & 0.450 & 0.084 \\
$95 \mathrm{~mm} \mathrm{Al}$ & 0.3587 & 0.089 & 0.410 & 0.083 \\
$150 \mathrm{~mm} \mathrm{Al}$ & 0.231 & 0.085 & 0.264 & 0.082 \\
$240 \mathrm{~mm} \mathrm{Al}$ & 0.160 & 0.079 & 0.160 & 0.079 \\
Desconcido & 0.210 & 0.075 & 0.21 & 0.075 \\
\hline
\end{tabular}

\section{ACKNOWLEDGMENT}

The authors would like to than EDP Group for their support during the development of this research, specially to Óscar Álvarez Pérez and José Manuel Carou Álvarez, without their constant support unraveling the raw data coming from GIS system, this work wouldn't have been possible.

\section{DATA AVAILABILITY}

Datasets related to this article can be found at http://dx.doi.org/10.17632/685vgp64sm.1, an open-source online data repository hosted at Mendeley Data [24].

\section{REFERENCES}

[1] "IEEE PES Distribution System Analysis Subcommittee's Distribution Test Feeder Working
Group.” [Online]. Available: http://sites.ieee.org/ pes-testfeeders/resources/

[2] European Statistical Office (EUROSTAT), "Urban Europe - statistics on cities, towns and suburbs executive summary," 2014.

[3] The European Committee for Electrotechincal Standardization, (CENELEC), "standards series hd 60364 - low voltage electrical installations," 2014.

[4] W. H. Kersting, "Radial distribution test feeders," IEEE Transactions on Power Systems, vol. 6, no. 3, pp. 975985, 1991.

[5] — "Radial distribution test feeders," in 2001 IEEE Power Engineering Society Winter Meeting. Conference Proceedings, vol. 2, 2001, pp. 908-912 vol.2.

[6] R. C. Dugan, W. H. Kersting, S. Carneiro, R. F. Arritt, and T. E. McDermott, "Roadmap for the IEEE PES test feeders," in 2009 IEEE/PES Power Systems Conference and Exposition, 2009, pp. 1-4.

[7] W. G. Sunderman, R. C. Dugan, and D. S. Dorr, "The neutral-to-earth voltage (NEV) test case and distribution system analysis," in 2008 IEEE Power and Energy Society General Meeting - Conversion and Delivery of Electrical Energy in the 21st Century, 2008, pp. 1-6.

[8] R. F. Arritt and R. C. Dugan, "The IEEE 8500-node test feeder," in IEEE PES T D 2010, 2010, pp. 1-6.

[9] W. H. Kersting, "A comprehensive distribution test feeder," in IEEE PES T\&D 2010, April 2010, pp. 1-4.

[10] K. Schneider, P. Phanivong, and J. S. Lacroix, "IEEE 342-node low voltage networked test system," in 2014 IEEE PES General Meeting - Conference Exposition, 2014, pp. 1-5.

[11] K. P. Schneider, Y. Chen, D. Engle, and D. Chassin, "A taxonomy of north american radial distribution feeders," in 2009 IEEE Power Energy Society General Meeting, 2009, pp. 1-6.

[12] IEEE PES AMPS DSAS Test Feeder Working Group, "IEEE PES distribution systems analysis subcommittee radial test feeders.” 2016. [Online]. Available: http: //sites.ieee.org/pes-testfeeders/

[13] Y. Li and P. J. Wolfs, "Taxonomic description for western australian distribution medium-voltage and low-voltage feeders," IET Generation, Transmission Distribution, vol. 8, no. 1, pp. 104-113, 2014.

[14] V. Rigoni, L. F. Ochoa, G. Chicco, A. Navarro-Espinosa, and T. Gozel, "Representative residential LV feeders: A case study for the north west of England," IEEE Transactions on Power Systems, vol. 31, no. 1, pp. 348360, 2016.

[15] C. Mateo, G. Prettico, T. Gómez, R. Cossent, F. Gangale, P. Frías, and G. Fulli, "European representative electricity distribution networks," International Journal of Electrical Power Energy Systems, vol. 99, pp. 273 280, 2018.

[16] W. H. Kersting, Distribution System Modeling and Analysis, ser. 3rd ed. New York NY: CRC Press, 2012.

[17] P. Arboleya, A. Koirala, L. Suárez-Ramón, B. Mohamed, and C. González-Morán, "Impact evaluation of the new self-consumption spanish scenario on the low voltage 
terminal distribution network," IEEE Transactions on Industry Applications, pp. 1-1, 2019.

[18] A. Sendin, T. Arzuaga, I. Urrutia, I. Berganza, A. Fernandez, L. Marron, A. Llano, and A. Arzuaga, "Adaptation of powerline communications-based smart metering deployments to the requirements of smart grids," Energies, vol. 8, no. 12, pp. 13 481-13 507, 2015.

[19] P. Arboleya, "State estimation in low voltage networks using smart meters: Statistical analysis of the errors," in 2018 IEEE Power Energy Society General Meeting (PESGM), Aug 2018, pp. 1-5.

[20] ADRES-CONCEPT, “(EZ-IF: Development of concepts for ADRES autonomous decentralized regenerative energy systems, project no. 815674). the project was funded by the austrian climate and energy fund and performed under the program ENERGIE DER ZUKUNFT." 2010.

[21] F. E. Postigo Marcos, C. Mateo Domingo, T. Gómez San Román, B. Palmintier, B.-M. Hodge, V. Krishnan, F. de Cuadra García, and B. Mather, "A review of power distribution test feeders in the united states and the need for synthetic representative networks," Energies, vol. 10, no. 11, 2017.

[22] R. C. Dugan, "Reference Guide; The Open Distribution System Simulator (OpenDSS)," EPRI (Electric Power Research Institute), User Manual, 2016. [Online]. Available: https://github.com/tshort/OpenDSS/ blob/master/Distrib/Doc/OpenDSSManual.pdf

[23] A. Prostejovsky, O. Gehrke, M. Marinelli, and M. Uslar, "Reduction of topological connectivity information in electric power grids," in 2016 51st International Universities Power Engineering Conference (UPEC), 2016, pp. 1-6.

[24] P. Arboleya, A. Koirala, L. Suárez-Ramón, and B. Mohamed, "Non-synthetic european low voltage test system," http://dx.doi.org/10.17632/685vgp64sm.1.

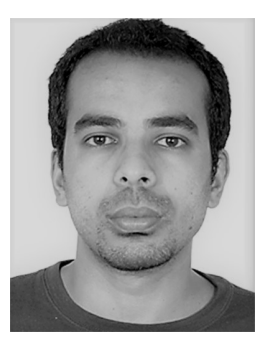

Arpan Koirala received his Master degree (2018) in Erasmus Mundus Master Course in Sustainable Transportation and Electrical Power Systems from consortium of University of Oviedo, Spain, La Sapienza, Rome, University of Nottingham, UK and Polytechnic Institute of Coimbra. He is currently pursuing his $\mathrm{PhD}$ degree from ESAT-ELECTA department of KU Leuven situated in Energyville, Genk. His areas of interest are distribution system modeling, energy market and high scale integration of renewable resources.

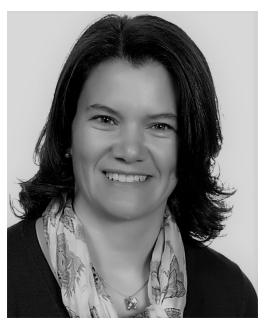

Lucía Suárez-Ramón received the M.Sc. degree in Electrical Engineering from the University of Oviedo, Gijón, Spain, in 1997. Nowadays, she works as Chief of Telemanagement System Infrastructure at EDP and she is pursuing her $\mathrm{PhD}$. at LEMUR Research Group at University of Oviedo. Her main research interests have to do with the smart operation of terminal distribution networks, networks analysis and simulation and failures detection.

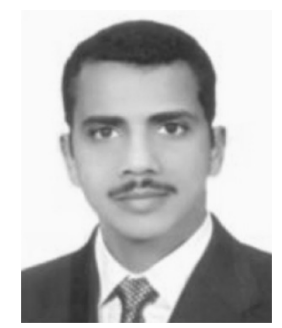

Bassam Mohamed received the M.Sc and Ph.D. degrees from the University of Oviedo, Gijón, Spain, in 2014 and 2018 respectively. He is now responsible of railway software development at LEMUR Research Group. His field of expertise has to do with the efficient development and implementation of algorithms for power systems analysis, specially those related to railway networks and unbalanced micro-grids. During the last years we developed several commercial software packages for electric network analysis and simulation.

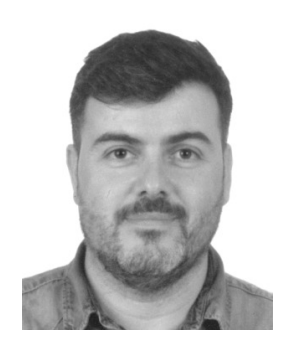

Pablo Arboleya (SM'13) received the M.Sc and Ph.D. (with distinction) degrees from the University of Oviedo, Gijón, Spain, in 2001 and 2005, respectively, both in Electrical Engineering. Nowadays, he works as Associate Professor in the Department of Electrical Engineering at the University of Oviedo, he is Managing Editor of the ELSEVIER's International Journal of Electrical Power \& Energy Systems and holder of the Gijón Smart Cities Chair at the University of Oviedo. Presently his main research interests are focused in the micro-grid and smart-grid modeling and operation techniques, Energy Internet applications and railway traction networks modelling and simulation. 